\title{
ADESÃO ÀS MEDIDÁS PARA PREVENÇÃO DA INFECÇÃO DA CORRENTE SANGUÍNEA RELACIONADA AO CATETER VENOSO CENTRAL
}

Alanna Gomes da Silva ${ }^{1}$, Adriana Cristina de Oliveira ${ }^{2}$

Objetivo: verificar a adesão da equipe multiprofissional para as medidas de prevenção da infecção da corrente sanguínea relacionada ao cateter venoso central. Metodologia: estudo quase-experimental, realizado na unidade de terapia intensiva de um hospital de urgência e emergência. A população foi composta pelas equipes médicas e de enfermagem. A coleta de dados ocorreu em três fases distintas: Período pré-intervenção, Período de intervenção e Período pós-intervenção. As análises foram por meio de estatística descritiva e pelos testes $\chi^{2}$ de Pearson e exato de Fisher. Resultados: verificou-se uma baixa adesão global pelos enfermeiros e técnicos, especialmente à higiene das mãos (22,7\%) e desinfecção do hub (10,4\%) e uma adesão de 100\% da equipe médica ao utilizar a barreira máxima de precaução. Conclusão: mesmo com um resultado satisfatório para a inserção dos cateteres nos períodos pré e pós-intervenção, essas medidas não representam completa adesão pela equipe de enfermagem.

Descritores: Infecções Relacionadas a Cateter, Unidades de Terapia Intensiva, Cateteres Venosos Centrais.

\section{ADHERENCE TO MEASURES TO PREVENT BLOODSTREAM INFECTION RELATED TO THE CENTRAL VENOUS CATHETER}

Objective: To verify the multiprofessional staff adherence to measures to prevent bloodstream infection related to the central venous catheter. Methodology: A quasi-experimental study, carried out at an intensive care unit from emergency and urgency hospital. The population was composed by medical and nursing staff. Data collection took place in three distinct phases: Preintervention period, intervention period and post-intervention period. Analysis were made through descriptive statistics and Pearson's $\chi^{2}$ and Fisher's exact tests. Results: There was a low overall adherence by nurses and technicians, especially to hand hygiene (22.7\%) and hub disinfection (10.4\%), and a 100\% adherence from medical staff when using the maximum barrier of precaution. Conclusion: Even with a satisfactory result for catheters insertion in the pre- and post-intervention periods, these measures are not enough when the catheter maintenance measures are not fully adhered by the nursing team.

Descriptors: Catheter-Related Infections, Intensive Care Units, Central Venous Catheters.

\section{ADHESIÓN PARA LA PREVENCIÓN DE INFECCIÓN DEL TORRENTE SANGUÍNEO RELACIONADAS CON EL CATÉTER VENOSO CENTRAL}

Objetivo: Determinar la adhesión de miembros del equipo multiprofesional para la prevención de infección del torrente sanguíneo relacionadas con el catéter venoso central. Metodología: Estudio cuasi-experimental realizado en una unidad de cuidados intensivos de un hospital de emergencia y urgencia. La población estaba compuesta por el personal médico y de enfermería. La recolección de datos ocurrió en tres fases: periodo previo a la intervención; Período de intervención y después de la intervención. Los análisis fueron hechos por la estadística descriptiva y las pruebas de $\chi^{2}$ de Pearson y la prueba exacta de Fisher. Resultados: Se observó una baja de miembros en general por las enfermeras y los técnicos, especialmente higiene de las manos (22,7\%) y la desinfección del hub (10,4\%) y una membresía de $100 \%$ del personal médico usando la barrera máximo precaución. Conclusión: Incluso con un resultado satisfactorio para la inserción de catéteres en el pre y post-intervención, estas medidas no son suficientes cuando las medidas para el mantenimiento de los catéteres no se cumplen por completo por el personal de enfermería.

Descriptores: Infecciones Relacionadas con Catéteres, Unidades de Cuidados Intensivos, Catéteres Venosos Centrales.

1Enfermeira.Mestre em Enfermagem. Universidade Federal de Minas Gerais-UFMG. E-mail: alannagomessilva@gmail.com ${ }^{2}$ Enfermeira. Doutora em Enfermagem. Professora Associada da Escola de Enfermagem da UFMG. 


\section{INTRODUÇÃO}

As infecções relacionadas à assistência à saúde (IRAS) são definidas como uma condição local ou sistêmica resultante de uma reação adversa à presença de um agente infeccioso ou sua toxina, podendo se manifestar a partir de 48 horas após a internação ${ }^{(1)}$.

Entre as IRAS mais frequentes está a infecção da corrente sanguínea e constitui umas das complicações relacionadas ao cateter venoso central (CVC) mais frequentes, dispendiosas, potencialmente letais, associando-se à elevada morbidade, com prolongamento do tempo de aumento dos custos de internação(2)

No Brasil, dados referentes a 2015 reportam uma taxa de infecção da corrente sanguínea relacionada ao CVC em UTI de 5,1 para 1000 cateteres dia(3); na Europa, 13,3 infecções para 1.000 cateteres dia $^{(4)}$ e, nos Estados Unidos, estima-se em torno de 30.000 novos casos dessa infecção por ano(5) ${ }^{(5)}$ taxa de mortalidade por infecção relacionada ao CVC pode atingir até $69 \%$ (2)

A redução das taxas de infecção requer uma abordagem global e multidisciplinar, incluindo intervenções comportamentais e educacionais de toda equipe envolvida na inserção e manutenção do CVC. O cumprimento das diretrizes é um passo essencial para melhoria das práticas de cuidado ao paciente em uso do $\mathrm{CVC}^{(6)}$.

Nesse contexto, monitorar a adesão às medidas para prevenção das infecções da corrente sanguínea relacionadas ao CVC dos profissionais pode ser uma estratégia que aponte lacunas e subsidie investimentos na melhoria das práticas assistenciais fundamentadas em treinamentos, educação continuada e feedback às equipes.

Com base no exposto, objetivou-se verificar a adesão da equipe multiprofissional às medidas para prevenção das infecções da corrente sanguínea relacionada ao CVC.

\section{METODOLOGIA}

Tratou-se de um estudo quase-experimental, realizado em uma UTI de 48 leitos de um hospital público de urgência e emergência de grande porte de Belo Horizonte, Minas Gerais, conduzido entre os meses de julho a novembro de 2016, após aprovação do Comitê de Ética em Pesquisa (CAAE 53642016.1.0000.5149).

Foram elegíveis, como população de estudo, os profissionais que prestavam assistência direta ao paciente em uso do CVC (médicos, enfermeiros e técnicos de enfermagem), que concordassem participar do estudo após apresentação e assinatura do Termo de Consentimento Livre Esclarecido (TCLE). A coleta de dados ocorreu em três fases distintas:
Fase 1 - Periodo pré-intervenção: consistiu na observação direta das equipes médica e de enfermagem durante a inserção e manipulação do CVC. A observação foi feita por alunos de iniciação científica devidamente treinados, buscando amenizar o efeito Hawthorne. As sessões de observação foram distribuidas pelos turnos de trabalho diurno e noturno, de segunda a sexta-feira, com duração média de três horas por turno.

Fase 2 - Periodo de Intervenção: foi realizada uma intervenção lúdica por meio de um jogo de dados, contendo aspectos relacionados à inserção e à manutenção do CVC, sendo realizada pela própria pesquisadora. As intervenções ocorreram em grupo de três a seis profissionais. Aqueles que, por algum motivo, não conseguiram participar da intervenção, foram abordados em outra ocasião a fim de garantir que todos os profissionais tivessem recebido algum treinamento. Foram utilizados cartazes para comunicações visuais, afixados em pontos estratégicos do ambiente de trabalho para alertar os profissionais das medidas para prevenção da infecção da corrente sanguínea.

Fase 3 - Período pós-intervenção: objetivou avaliar o impacto das estratégias implementadas na fase 2 do estudo sobre a adesão às medidas para prevenção da infecção da corrente sanguínea relacionadas ao CVC. Foi realizado 60 dias após a intervenção, novamente conduzida por observação direta dos profissionais, utilizando o mesmo método e instrumento de observação da fase 1. No entanto, os dois alunos de iniciação científica que procederam essa observação foram diferentes daqueles que participaram da fase 1 .

As análises foram realizadas com o auxilio do programa estatístico Data Analysis and Statistical Software (Stata), versão 14, por estatística descritiva. Para a comparação dos dados, foi realizado teste de Pearson quando o valor esperado foi $>5$ teste exato de Fisher para o valor esperado $<5$. O nivel de significância considerado para inferência estatística foi de $5 \%(p=0,05)$ e o intervalo de confiança de $95 \%$

\section{RESULTADOS}

Os resultados deste estudo estão apresentados em consonância com as etapas da metodologia.

\section{Fase 1 - Periodo pré-intervenção}

Foram registradas 287 oportunidades ou indicações para prevenção das infecções da corrente sanguínea quanto aos cuidados durante a manutenção do CVC e a equipe médica foi observada em relação a oito inserções do CVC, sendo observadas oito inserções (Tabela 1). 
Tabela 1 - Observação direta da equipe multiprofissional em relação às medidas para prevenção da infecção da corrente sanguínea relacionada ao CVC no período pré-intervenção. Belo Horizonte, 2016

$\begin{array}{ll}\text { Administração de } & \text { Oportunidades } \\ \text { medicamentos pelo CVC } & \text { globais = 192 } \\ \text { (Técnicos de Enfermagem) } & n(\%)\end{array}$

Higiene das mãos antes de administrar medicamentos

(48 oportunidades)

Higiene simples

Desinfecção do hub

(48 oportunidades)

Uso de luva de procedimento

$5(10,4)$

(48 oportunidades)

Higiene das mãos após administrar medicamentos (48 oportunidades)

Higiene simples

$11(22,9)$

Fricção Alcóolica

$2(4,2)$

Manipulação do curativo do CVC (Técnicos de Enfermagem)

Oportunidades globais $=39$ $n(\%)$

Higiene das mãos antes de manipular o curativo (13 oportunidades)

Higiene simples

Uso de luva de procedimento

$10(76,9)$

(13 oportunidades)

Higiene das mãos após manipular o curativo (13 oportunidades)

Higiene simples

$5(38,5)$

Troca do curativo do CVC (Enfermeiros)

Oportunidades globais $=\mathbf{5 6}$ $n(\%)$

Higiene das mãos antes de trocar o curativo (14 oportunidades)

Higiene simples

Uso de luva estéril

$11(78,6)$

(14 oportunidades)

Assepsia da pele do paciente

$14(100)$

(14 oportunidades)

Higiene das mãos após trocar o

curativo (14 oportunidades)

Higiene simples

Fricção Alcóolica

Inserção do CVC

Oportunidades $=8$

(Médicos)

$n(\%)$

Preparo cirúrgico das mãos

$8(100)$

Uso da barreira máxima de precaução

8 (100)

Assepsia da pele do paciente

Higiene das mãos após inserção

Fricção Alcóolica

$4(50)$
Observou-se uma baixa adesão às medidas para prevenção das infecções da corrente sanguinea relacionadas ao CVC. Das oportunidades observadas para higiene das mãos, a taxa de adesão total foi de 22,7\% e, dessas, $20 \%$ foram higiene simples e 2,7\% fricção alcóolica.

\section{Fase 2 - Período de Intervenção}

Nessa fase, ocorreu o treinamento da equipe multiprofissional em relação às medidas para prevenção da infecção da corrente sanguínea relacionada ao CVC. Participaram 138 funcionários. Desses, 24 (17,4\%) eram médicos, 19 (13,8\%) enfermeiros e 95 (68,8\%) técnicos de enfermagem. $\bigcirc$ jogo de dados teve uma duração média de 25 minutos e foi realizado em grupos de três a seis profissionais, totalizando 41 sessões. Foram abordadas, durante a intervenção educativa, as medidas para prevenção da infecção no momento da inserção e manutenção do CVC.

\section{Fase 3 - Periodo pós-intervenção}

Após a intervenção, novamente, foram realizadas observações diretas da equipe médica e de enfermagem, totalizando 98 horas de observação, registrando-se 484 oportunidades para prevenção das infecções da corrente sanguínea quanto aos cuidados durante a manutenção do CVC. Pela equipe médica, foram observadas 10 inserções de CVC (Tabela 2).

Tabela 2 - Observação direta da equipe multiprofissional em relação às medidas para prevenção da infecção da corrente sanguínea relacionada ao CVC no período pós-intervenção. Belo Horizonte, 2016

\begin{tabular}{|c|c|}
\hline $\begin{array}{l}\text { Administração de medicamentos pelo CVC } \\
\text { (Técnicos de enfermagem) }\end{array}$ & $\begin{array}{l}\text { Oportunidades } \\
\text { globais = } 344 \\
n(\%)\end{array}$ \\
\hline \multicolumn{2}{|l|}{$\begin{array}{l}\text { Higiene das mãos antes de administrar } \\
\text { medicamentos ( } 86 \text { oportunidades) }\end{array}$} \\
\hline Higiene simples & $3(3,5)$ \\
\hline Fricção Alcóolica & $15(17,4)$ \\
\hline $\begin{array}{l}\text { Uso de luvas de procedimento } \\
\text { ( } 86 \text { oportunidades) }\end{array}$ & $34(39,5)$ \\
\hline Desinfecção do hub (86 oportunidades) & $11(12,8)$ \\
\hline \multicolumn{2}{|l|}{$\begin{array}{l}\text { Higiene das mãos após administrar } \\
\text { medicamentos ( } 86 \text { oportunidades) }\end{array}$} \\
\hline Higiene simples & $6(7)$ \\
\hline Fricção Alcóolica & $13(15,1)$ \\
\hline $\begin{array}{l}\text { Manipulação do curativo do CVC } \\
\text { (Técnicos de enfermagem) }\end{array}$ & $\begin{array}{l}\text { Oportunidades } \\
\text { globais }=36 n(\%)\end{array}$ \\
\hline
\end{tabular}

Higiene das mãos antes de manipular o curativo (12 oportunidades)

Fricção Alcóolica 


\begin{tabular}{|c|c|}
\hline $\begin{array}{l}\text { Uso de luvas de procedimento } \\
\text { (12 oportunidades) }\end{array}$ & $7(58,3)$ \\
\hline \multicolumn{2}{|l|}{$\begin{array}{l}\text { Higiene das mãos após manipular o } \\
\text { curativo ( } 12 \text { oportunidades) }\end{array}$} \\
\hline Higiene simples & $2(16,7)$ \\
\hline $\begin{array}{l}\text { Troca do curativo do CVC } \\
\text { (Enfermeiros) }\end{array}$ & $\begin{array}{l}\text { Oportunidades } \\
\text { globais = } 104 \\
n(\%)\end{array}$ \\
\hline \multicolumn{2}{|l|}{$\begin{array}{l}\text { Higiene das mãos antes de trocar o } \\
\text { curativo ( } 26 \text { oportunidades) }\end{array}$} \\
\hline Higiene simples & $6(23,1)$ \\
\hline Fricção Alcóolica & $4(15,4)$ \\
\hline Uso de luva estéril (26 oportunidades) & $26(100)$ \\
\hline $\begin{array}{l}\text { Assepsia da pele do paciente } \\
\text { ( } 26 \text { oportunidades) }\end{array}$ & $26(100)$ \\
\hline \multicolumn{2}{|l|}{$\begin{array}{l}\text { Higiene das mãos após trocar o curativo } \\
\text { ( } 26 \text { oportunidades) }\end{array}$} \\
\hline Higiene simples & $6(23,1)$ \\
\hline Fricção Alcóolica & $1(3,8)$ \\
\hline $\begin{array}{l}\text { Inserção do CVC } \\
\text { (médicos) }\end{array}$ & $\begin{array}{l}\text { Oportunidades }=10 \\
(\%)\end{array}$ \\
\hline Preparo cirúrgico das mãos & $10(100)$ \\
\hline Uso da barreira máxima de precaução & $10(100)$ \\
\hline Assepsia da pele do paciente & $10(100)$ \\
\hline \multicolumn{2}{|l|}{ Higiene das mãos após inserção } \\
\hline Higiene simples & $3(30)$ \\
\hline Fricção Alcóolica & $3(30)$ \\
\hline
\end{tabular}

Nessa fase, observou-se uma baixa adesão às medidas para prevenção das infecções da corrente sanguínea relacionadas ao CVC, especialmente no que se refere à administração de medicamentos pelo CVC e manipulação do curativo.

A comparação das observações diretas dos profissionais nos períodos pré e pós-intervenção está apresentada na Tabela 3.

Tabela 3 - Comparação das observações diretas dos profissionais nos periodos pré e pós-intervenção. Belo Horizonte, 2016

$\begin{array}{llll}\begin{array}{l}\text { Administração de } \\ \text { medicamentos pelo } \\ \text { CVC }\end{array} & \begin{array}{l}\text { Pré- } \\ \text { intervenção } \\ n(\%)\end{array} & \begin{array}{l}\text { Pós- } \\ \text { intervenção } \\ \boldsymbol{n}(\%)\end{array} & \begin{array}{l}\text { Valor } \\ \boldsymbol{p}\end{array} \\ \begin{array}{l}\text { Higiene das mãos } \\ \text { antes de administrar } \\ \text { medicamentos }\end{array} & 1(2,1) & 18(20,9) & 0.003 \\ \text { Sim } & 47(98,9) & 68(79,1) & \\ \text { Não } & & & \\ \begin{array}{l}\text { Uso de luvas de } \\ \text { procedimento }\end{array} & 14(29,2) & 34(39,5) & 0.230 \\ \text { Sim } & 34(70,8) & 52(60,5) & \\ \text { Não } & & & \end{array}$

Desinfecção do hub
$\begin{array}{llll}\text { Sim } & 5(10,4) & 11(12,8) & 0.684 \\ \text { Não } & 43(89,6) & 75(87,2) & \end{array}$

Higiene das mãos após administrar medicamentos

$\begin{array}{llll}\text { Sim } & 13(27,1) & 19(22,1) & 0.516 \\ \text { Não } & 35(72,9) & 67(77,9) & \\ \begin{array}{l}\text { Manipulação do } \\ \text { curativo do CVC }\end{array} & \begin{array}{l}\text { Pré- } \\ \text { intervenção } \\ \text { n(\%) }\end{array} & \begin{array}{l}\text { Pós- } \\ \text { interven- } \\ \text { ção }\end{array} & \begin{array}{l}\text { Valor } \\ \mathbf{n ( \% )}\end{array} \\ & & \mathbf{n} & \end{array}$

Higiene das mãos antes de manipular o curativo

$\begin{array}{llll}\text { Sim } & 1(7,7) & 3(25) & 0.322 \\ \text { Não } & 12(92,3) & 9(75) & \\ \begin{array}{l}\text { Uso de luvas de } \\ \text { procedimento }\end{array} & & & \\ \text { Sim } & 10(76,9) & 7(58,3) & 0.319 \\ \text { Não } & 3(23,1) & 5(41,7) & \end{array}$

Higiene das mãos após manipular o curativo

$\begin{array}{llll}\text { Sim } & 5(38,5) & 2(16,7) & 0.378 \\ \text { Não } & 8(61,5) & 10(83,3) & \\ \begin{array}{l}\text { Troca do curativo } \\ \text { do CVC }\end{array} & \begin{array}{l}\text { Pré- } \\ \text { intervenção } \\ n(\%)\end{array} & \begin{array}{l}\text { Pós- } \\ \text { interven- } \\ \text { ção } \\ n(\%)\end{array} & \begin{array}{l}\text { Valor } \\ \text { n(\%) }\end{array} \\ & & & \end{array}$

Higiene das mãos antes de trocar o curativo

$\begin{array}{llll}\text { Sim } & 6(42,9) & 10(38,5) & 0.787 \\ \text { Não } & 8(57,1) & 16(61,5) & \\ \text { Uso de luva estéril } & & & \\ \text { Sim } & 11(78,6) & 26(100) & 0.014 \\ \text { Não } & 3(21,4) & 0 & \end{array}$

Higiene das mãos após trocar o curativo

$\begin{array}{llll}\text { Sim } & 8(57,1) & 7(26,9) & 0.060 \\ \text { Não } & 6(42,9) & 19(73,1) & \\ \text { Inserção do CVC } & \begin{array}{l}\text { Pré- } \\ \text { intervenção } \\ \text { n(\%) }\end{array} & \begin{array}{l}\text { Pós- } \\ \text { interven- } \\ \text { ção }\end{array} & \begin{array}{l}\text { Valor } \\ \text { n(\%) }\end{array} \\ & & & \end{array}$

Higiene das mãos

após inserção

$\begin{array}{llll}\text { Sim } & 4(50) & 6(60) & 1.000 \\ \text { Não } & 4(50) & 4(40)\end{array}$


Observou-se um aumento da adesão dos profissionais após a intervenção educativa entre as variáveis higiene das mãos antes de administrar medicamentos e uso de luvas na troca do curativo $(p<0,05)$.

\section{DISCUSSÃO}

A observação direta dos profissionais apontou para uma baixa adesão à higiene das mãos antes e após a administração de medicamentos, manipulação e troca do curativo, bem como no uso de luvas e desinfecção do hub, independente das fases do estudo.

A higienização das mãos é reconhecida como a prática mais efetiva na prevenção das IRAS. No entanto, apesar da sua importância, a adesão a essa prática permanece baixa nos serviços de saúde, com taxas mundiais de adesão de aproximadamente $38,7 \%{ }^{(7)}$. Alguns estudos também confirmam esse resultado: uma revisão sistemática que incluiu 65 estudos realizados em UTI,

a taxa média de adesão foi de $40 \%{ }^{(8)}$. Em 446 oportunidades para higienização das mãos, a adesão dos profissionais em uma UTI no Brasil foi de $43,7 \%{ }^{(9)}$. Com o objetivo de avaliar a adesão à higienização das mãos na prevenção de infecção da corrente sanguínea relacionada ao CVC, foi observado que a maioria dos profissionais não realizou a higienização das mãos antes e após as seguintes práticas avaliadas: troca do sistema de infusão (89,7\%), administração de medicamentos $(72,9 \%)$, troca e realização de curativo $(73,1 \%)^{(10)}$. Quando a prática de higiene das mãos não acontece adequadamente favorece a transmissão cruzada de microrganismos, principalmente em pacientes críticos que apresentam maior possibilidade de serem colonizados ou infectados.

O uso de luvas entre os profissionais, para a realização dos procedimentos, foi maior que a higiene das mãos. Em estudo que avaliou a relação do uso de luvas com a adesão à higiene de mãos, foi observada menor higiene das mãos quando os profissionais faziam uso de luvas ${ }^{(11)}$. De forma semelhante em outro estudo, foi observado que de 1.067 oportunidades de higienização das mãos, essa ocorreu em apenas 14,8\% antes de usar luvas e, após a retirada das luvas, houve um aumento para 56,6\% (12). Desse modo, percebe-se que a higienização das mãos pode ser menos frequente quando o profissional faz uso de luvas.
A adesão à desinfecção do hub antes de administrar medicamentos pelo CVC também foi baixa. Em um estudo que avaliou as práticas de prevenção e controle de infecção da corrente sanguínea, foi constatado que, de todas as medidas observadas durante a manipulação do cateter, a desinfecção do hub apresentou menor adesão (40\%)(13). Nos Estados Unidos, foi implementado um bundle de cuidados para o hub do cateter, sendo obtida, após sete meses de treinamento, uma adesão de $99 \%$ à desinfecção do hub antes da realização de medicamentos. Além disso, verificaram uma redução significativa em casos de sepse por Gram-negativo(14). Os resultados reafirmam a importância do treinamento e educação continuada da equipe responsável pela manipulação do cateter, principalmente em relação a desinfecção do hub, visto que muitos profissionais desconhecem a técnica e sua finalidade.

Ressalta-se que, para inserção do CVC, a adesão ao preparo cirúrgico das mãos, uso da barreira máxima de precaução e assepsia da pele do paciente foram de $100 \%$ nos períodos pré e pós-intervenção. Tais resultados demonstram que a equipe médica seguiu corretamente $\mathrm{O}$ uso das medidas recomendadas para prevenção das infecções da corrente sanguínea durante a inserção do CVC.

No presente estudo, a intervenção educativa foi por meio de uma atividade lúdica com um jogo de dados, sendo uma proposta de treinamento lúdico, justificado pelo fato de que os treinamentos devem ser dinâmicos e voltados, principalmente, para a sensibilização do profissional, com foco em normas e atitudes e não apenas na transmissão de informações. A educação aumenta o conhecimento, mas não necessariamente melhora a prática assistencial, não existindo uma associação direta de que a falta de conhecimento da equipe afeta o cumprimento das diretrizes ${ }^{(15)}$. Assim, o conhecimento é diferente da prática real do cotidiano, pois as pessoas agem de acordo com suas intenções e percepções, inspiradas por atitudes em relação ao comportamento e normas subjetivas ${ }^{(16)}$.

Quanto às limitações, pode-se apontar o seguimento prospectivo, devido às perdas, por férias e licenças saúde, durante as etapas da coleta de dados. $O$ curto período para realização do estudo foi impeditivo para executar a etapa de educação continuada e analisar o seu impacto em longo prazo. 


\section{CONCLUSÃO}

Durante a observação direta dos profissionais, a higiene das mãos antes e após a realização dos procedimentos e a desinfecção do hub antes de administrar medicamentos não foram aderidas complemente pelos enfermeiros e técnicos de enfermagem. Por outro lado, observou-se maior adesão da equipe médica ao utilizar todas as medidas de barreira que são preconizadas durante a inserção do CVC, tanto no periodo pré-intervenção, quanto no pós-intervenção.

Diante dos achados, se reforça a importância de um maior investimento na discussão da prevenção da infecção da corrente sanguínea, bem como na educação continuada das equipes em suas fragilidades demonstradas no presente estudo, especialmente às medidas de manutenção do CVC.

\section{REFERÊNCIAS}

1. World Health Organization. Report on the Burden of Endemic Health Care-Associated Infection Worldwide. [Internet] 2011 [cited 2017 Jan 10]; Available from: http://apps.who.int/iris/ bitstream/10665/80135/1/9789241501507_eng.pdf

2. Gahlot R,Nigam C, Kumar V, Yadav G, Anupurba S. Catheter-related bloodstream infections. Int J Crit Illn Inj Sci [Internet] 2014 [cited 2017 Jan 10]; 4(2):162-167. Available from: https://www.ncbi.nlm.nih. gov/pmc/articles/PMC4093967/

3. Agência Nacional de Vigilância Sanitária. Avaliação dos indicadores nacionais de infecção relacionada à assistência ano de 2014 e relatório de progresso. Brasilia. 2015;6(11):1-37.

4. Mok Q. How I diagnose and manage catheter-associated blood stream infections.

J Paediatr Child Health [Internet] 2015 [cited 2017 Jan 10]; 25(5):243244. 4(2):162-167. Available from: https://www.ncbi.nlm.nih.gov/pmc/ articles/PMC4093967/

5. National Healthcare Safety Network. Patient Safety Component Manual. 2017 [cited 2017 Jan 5]. Available from: https://www.cdc.gov/ nhsn/pdfs/pscmanual/pcsmanual_current.pdf

6. Agência Nacional de Vigilância Sanitária. Programa nacional de prevenção e controle de infecções relacionadas à assistência à saúde (2016-2020). Brasilia. 2016:1:1-38

7. World Health Organization. WHO guidelines on hand hygiene in health care- First Global Patient Safety Challenge Clean Care is Safer Care. Geneva [Internet] 2009 [cited 2017 Jan 10]. Available from http://apps.who.int/iris/bitstream/10665/44102/1/9789241597906_ eng.pdf

8. Erasmus V, Daha TJ, Brug H, Richardus JH, Behrendt MD, Vos MC et al. Systematic review of studies on compliance with hand hygiene guidelines in hospital care. Infect Control Hosp Epidemiol [Internet] 2010 [cited 2017 Jan 10]; 31(3): 283-294. Available from: https://www. ncbi.nlm.nih.gov/pubmed/20088678

9. Souza LM, Ramos MF, Becker ESS, Meirelles LCS, Monteiro SAO. Adesão dos profissionais de terapia intensiva aos cinco momentos da higienização das mãos. Rev Gaucha Enferm [Internet] 2015 [cited
2017 Dec 5]; 36(4): 21-28. Available from: http://www.scielo.br/scielo. php?pid=S1983-14472015000400021Escript=sci_arttextEtlng=pt

10. Oliveira FJG, Caetano JA, Silva VM, Almeida PC, Rodrigues AB, Siqueira JF. O uso de indicadores clínicos na avaliação das práticas de prevenção e controle de infecção de corrente sanguinea. Texto contexto enferm [Internet] 2015 [cited 2017 Dec 5]; 24(4): 10181026. Available from: http://www.scielo.br/scielo.php?pid=SO10407072015000401018\&script=sci_arttext\&tlng=pt

11. Fuller C, Savage J, Besser S, Hayward A, Cookson B, Cooper B et al. "The dirty hand in the latex glove": a study of hand hygiene compliance when gloves are worn. Infect Control Hosp Epidemiol [Internet] 2011 [cited 2017 Dec 5]; 32(12): 1194-1199. Available from: http://www.abih. net.br/wp-content/uploads/Fuller-et-al-_-ICHE-Dez2011.pdf

12. Ghorbani A, Sadeghi L, Shahrokhi A, Mohammadpour A, Addo M. Khodadadi E. Hand hygiene compliance before and after wearing gloves among intensive care unit nurses in Iran. Am J Infect Control [Internet] 2016 [cited 2017 Dec 5]; 44(11): 279-281. Available from: http:// www.sciencedirect.com/science/article/pii/S0196655316304175

13. Jardim JM, Lacerda RA, Soares NJD, Nunes BK. Avaliação das práticas de prevenção e controle de infecção da corrente sanguínea em um hospital governamental. Rev. esc. enferm. USP [Internet] 2013 [cited 2017 Mar 20]; 47(1): 38-45. Available from: http://www.scielo.br/ scielo.php?script=sci_arttextEpid=\$0080-62342013000100005

14. Sannoh S, Clones B, Munoz J, Montecalvo M, Parvez B. A multimodal approach to central venous catheter hub care can decrease catheterrelated bloodstream infection. Am J Infect Control [Internet] 2010 [cited 2017 Feb 14]; 36(8): 424- 429. Available from: http://www. sciencedirect.com/science/article/pii/S0196655309009493

15. Ward V, Smith S, House A, Hamer S. Exploring knowledge exchange: a useful framework for practice and policy. Soc Sci Med [Internet] 2012 [cited 2017 Feb 14];74(3):297-304.. Available from: http://www. sciencedirect.com/science/article/pii/S0277953611005922

16. Comer A, Harris AD, Shardell M, Braun B, Belton BM, Wolfsthal, SD et al. Web-Based Training Improves Knowledge about Central Line Bloodstream Infections. Infection Control and Hospital Epidemiology [Internet] 2011[cited 2017 Jan 12]; 32(12):1219-1222. Available from: https://www.ncbi.nlm.nih.gov/pmc/articles/PMC3331708/ 